\title{
昉Digital Publisher
}

ISSN 2588-0705

\section{Empatía y calidad de servicio. "Papel clave en las emociones positivas en equipos de Trabajo"}

Empathy and quality of service: "Key role in positive emotions in work Teams"

Carlos Eduardo Cervantes-Chan Instituto Tecnológico Superior de Escárcega - México 200205068@itsescarcega.edu.mx

Hugo Mauricio Lara-López Instituto Tecnológico Superior de Escárcega - México 200205035@itsescarcega.edu.mx

Geidy de los Ángeles Gómez-Xul Instituto Tecnológico Superior de Escárcega - México

geidy_gomez@itsescarcega.edu.mx doi.org/10.33386/593dp.2021.6-1.869

V6-N6-1 (dic) 2021, pp. 147-158 | Recibido: 11 de noviembre de 2021 - Aceptado: 09 de diciembre de 2021 (2 ronda rev.) Edición Especial 
Cómo citar este artículo:

APA:

Cervantes-Chan, C., \& Lara-López, H., \& Gómez-Xul, G., (2021).Empatía y calidad de servicio. "Papel clave en las emociones positivas en equipos de Trabajo". 593 Digital Publisher CEIT, 6(6-1), 147--158-. https://doi. org/10.33386/593dp.2021.6-1.869

Descargar para Mendeley y Zotero

\section{RESUMEN}

Esta investigación tiene como propósito analizar la relación de la dimensión de la empatía con la calidad de servicio que se le brinda al cliente con base en el modelo SERVQUAL en una tienda de abarrotes del municipio de Escárcega, Campeche. Es muy importante, que dentro de los equipos de trabajo que se forman en las empresas, se mantenga las emociones positivas para un desarrollo eficaz; la problemática con las empresas es que no ofrece un proceso de ayuda hacia las necesidades del cliente. El tipo de investigación es de tipo descriptivo cualitativo, basándose en datos obtenidos a través de la aplicación de un cuestionario a partir de la metodología SERVQUAL conformada por cinco dimensiones. Los resultados obtenidos con respecto a la empatía y las otras dimensiones es favorable; los consumidores tienen la percepción de la empresa, que proporciona los equipos adecuados al personal para el desempeño de sus funciones laborales, por el contrario, se encuentra como área de oportunidad de que el personal tenga un trato personalizado hacia sus clientes. Se concluye que la empatía proyecte de manera personal a los clientes, depende de la empatía que la empresa tenga para sus colaboradores, debiendo reforzar y orientar en el tema de las emociones, trabajo en equipo que logre apoyar en la mejora continua en la calidad en el servicio que oferte la empresa.

Palabras claves: empatía; calidad de servicio; emociones positivas; modelo SERVQUAL

\section{ABSTRACT}

The purpose of this research is to analyze the relationship of the dimension of empathy with the quality of service provided to the customer based on the SERVQUAL model in a grocery store in the municipality of Escárcega, Campeche. It is very important that within the work teams that are formed in companies, positive emotions are maintained for effective development; The problem with companies is that they do not offer a process to help the client's needs. The type of research is of a qualitative descriptive type, based on data obtained through the application of a questionnaire from the SERVQUAL methodology made up of five dimensions. The results obtained with respect to empathy and the other dimensions is favorable; Consumers have the perception of the company, which provides the personnel with adequate equipment for the performance of their work functions, on the contrary, it is found as an area of opportunity for the personnel to have a personalized treatment towards their clients. It is concluded that empathy projects clients personally, it depends on the empathy that the company has for its collaborators, having to reinforce and guide about emotions, teamwork that manages to support continuous improvement in quality in the service offered by the company.

Keywords: empathy; quality of service; positive emotions; SERVQUAL Model. 


\section{Introducción}

Menciona (Flores, 2012) la empatía es la que recuerda que las personas están hechas de la misma sustancia: de materia humana, de fibras emocionales, de ganas de vivir, amar y ser.

Si una empresa desea ser verdaderamente exitosa y progrese, debe contar con un equipo optimista y unido compuesto por colegas que se sientan en un ambiente de respeto y valor hacia su persona, para hacer lo mejor por la empresa. En contexto, si una persona valora a los demás, se considera empática, es otras palabras, aprecia lo que se habla, lo que hacen y cómo se sienten los demás. Escuchan atentamente y tratan de entender al otro individuo. Enciman, interactúan de manera abierta y honesta, mostrando un deseo real de establecer una conexión y un fuerte entendimiento entre ellos.

Según el informe (Businessolver, 2017), la empatía tiene un impacto directo en la productividad, la lealtad y el compromiso de los empleados.

"La gran complejidad del mundo laboral y la innovación generan diferentes situaciones que requieren diversidad de habilidades, altos niveles de conocimiento, respuestas rápidas $\mathrm{y}$ adaptabilidad. Y es a través de los equipos donde se pueden desarrollar todas estas características" (Kozlowski, 2006), esto quiere decir que entre más crecimiento y desarrollo tenga una empresa, más alta es la demanda de exigencia de los clientes, debido a los cambios en los gustos y preferencias del mercado; lo que genera que la organización desarrolle y genere estrategias diferenciadoras para distinguirse de la competencia.

Actualmente los problemas internos dentro de la institución son relevantes para su atención y afectan a toda la estructura, la falta de líderes empáticos dentro de una institución, desequilibran completamente la funcionalidad del personal de trabajo, ya que estos tratan de conectarse con su equipo, construyendo un sentido de confianza, apertura y comprensión que hace que los empleados se sientan apreciados, valorados, escuchados, reconocidos y cuidados. Empatía en el servicio al cliente es una herramienta eficaz para las organizaciones. Comprende las necesidades del cliente y las gestiona en el momento adecuado puede ayudarlo a evitar sentirse malogrado y llevarlo a estar conforme con el producto o servicio brindado por las empresas. El presente estudio contribuye a Comprender la relación entre la empatía en la calidad del servicio y las emociones positivas en el equipo de trabajo de la empresa, analizando las percepciones de empatía de los clientes en un estudio de caso de una empresa de abarrotes en Escárcega, Campeche. Desde una perspectiva empresarial, la empatía se considera clave en el modelo mencionado, ya que se trata de un constructo psicosocial, incluido en la "Calidad de Servicio" (Berry L. A., 1999) "El enriquecimiento de un servicio superior de calidad recae sobres todos provocando una reacción en cadena de los que se benefician ante el: consumidores, empleados, gerentes y accionista provocando en general $\mathrm{y}$ ende el bienestar de la comunidad. Como se percibe actualmente los negocios venden con base en el trato e imagen de la empresa, se enfocan más al trato que reciben al momento de consumir en los establecimientos, los seres humanos nos sentimos más en confianza cuando propician un contacto o sentir hacia la persona, $y$ lo mismo pasa en el trabajo en equipo "El trabajo en equipo es considerado un punto clave y una ventaja competitiva" (Roussea, 2006)

Se pretende buscar empleados saludables, con recursos psicológicos estables, que tengan la capacidad de dar un trato digno a los clientes, que tengan una conducta que beneficie a la empresa y que ésta sea realizada de manera voluntaria; las emociones positivas dan resultados organizacionales saludables por excelencia: como lo es la calidad de servicio" (Hernandez, 2014) lo que provoca un contagio mutuo con la persona. En concreto, probaremos el efecto mediador de las emociones positivas sobre la empatía en el trabajo en equipo, teniendo en cuenta las opiniones generales de los miembros del equipo de trabajo y las opiniones de los encargados directos sobre la calidad de los servicios de su equipo. 
Es importante fomentar la empatía en las empresas teniendo como base la integración de los grupos de trabajo. "Para poder realizar los diferentes procesos o llevar a cabo las diferentes tareas, se precisa de la colaboración y cooperación de varios miembros, estimulando de este modo la participación y la comunicación entre ellos y generando una mejora y un incremento de la calidad" (Cohen, 1997)

Según (Frdrickson, 2001), las emociones como el entusiasmo, la satisfacción y la alegría, comparten la propiedad de ampliar los repertorios de pensamiento y de acción de las personas $\mathrm{y}$ de construir reservas de recursos físicos, intelectuales, psicológicos y sociales disponibles para momentos futuros de crisis. Esta también es papel fundamental en el éxito de las empresas, más enfocado en el personal laboral (Perez, 2005) "Aborda el tema e indica que la empatía es el procedimiento en el que se abarcan todas las habilidades relacionadas con la comunicación entre el individuo y los sentimientos, sean propios o ajenos, también, se entiende como la capacidad cognitiva de distinguir en un contexto común lo que otro individuo puede sentir. Es un sentimiento de participación afectiva de una persona, en la realidad, que afecta a otra." Posteriormente los factores que rodean en el establecimiento (David, 2007)"Dice investigar lo que ellos le ofrecen a sus clientes en cuanto a variedad, precio, calidad de sus bienes y el servicio que les brindan, te permitir compararlo con lo que haces. Al saber esto, tendrás la oportunidad de detectar en que aspectos del negocio estas fallando, para realizar las adecuaciones necesarias. "que se labora, en el ámbito social y emocional, la conducta del mismo individuo a la hora de laborar o emprender un negocio que se base en las interacciones personales del hacia otra persona, la comunicación física y entablar una conexión con el cliente para que este consuma los productos o venda una buena proporción de lo que ofrece el local (Castillo, 2009)"

La empatía puede ser considerada como una de las virtudes más difíciles de aprender ya que implica ver más allá de sí mismo y a su vez poder preocuparse, sentirse y tratar de entender a las demás personas. Desde pequeño se le enseña al niño a poder llegar a tener la capacidad de ponerse en los zapatos de la otra persona para poder tener conciencia de los sentimientos y pensamientos de los demás aparte de sí mismo." En este contexto, se plantea la siguiente hipótesis: La empatía positiva, median parcialmente la relación entre emociones y calidad de servicio en el trabajo en equipo.

\section{Tabla 1}

Reflexiones de conceptos: empatía, emociones y equipos de trabajo

\begin{tabular}{|l|l|}
\hline AUTOR & \\
\hline (Hogan, 1969) & $\begin{array}{l}\text { "Definió la empatía como un intento de comprender } \\
\text { lo que pasa por la mente de los demás o, en otras pa- } \\
\text { labras, como la construcción que uno mismo tiene } \\
\text { que llevar a cabo sobre los estados mentales ajenos" }\end{array}$ \\
\hline (Bravo, 2016) & $\begin{array}{l}\text { "El uso correcto de estos elementos puede ofrecer } \\
\text { beneficios para las empresas, ya que la identidad vi- } \\
\text { sual y la comunicación interna ayudan a asociar a la } \\
\text { organización en las mentes de los usuarios y sentir } \\
\text { empatía por esta y sus ideales" }\end{array}$ \\
\hline (Gary, 2013) & $\begin{array}{l}\text { "La competencia de empatía proporciona a las per- } \\
\text { sonas conciencia de las emociones, preocupaciones } \\
\text { y necesidades de los demás. La empatía permite } \\
\text { leer corrientes emocionales. Esta sensibilidad en- } \\
\text { focada hacia los demás resulta crítica para el des- } \\
\text { empeño laboral cuando se concentra en la relación } \\
\text { con la gente" }\end{array}$ \\
\hline $\begin{array}{l}\text { (Garcia Prieto, } \\
2003)\end{array}$ & $\begin{array}{l}\text { "Los equipos de trabajo construyen y reconstruyen } \\
\text { su identidad de forma permanente en el hacer sien- } \\
\text { do por tanto artificial esta división entre ser y hacer" }\end{array}$ \\
\hline (Frdickson,, & $\begin{array}{l}\text { "Una de las teorías actuales en emociones y en la } \\
\text { cual centramos el foco de nuestra investigación es } \\
\text { nes positivas (ByB) " y construcción de emocio- }\end{array}$ \\
\hline
\end{tabular}

\section{Método}

Como investigación de campo, tipo cuantitativa - cualitativa elaborado en un tiempo promedio de una semana, enfocada dimensión de empatía para medir la calidad de servicio que se les brinda a los clientes en la empresa analizada, del giro comercial de abarrotes, se mencionan las fases que estructuran el estudio:

1. Establecer el modelo de medición de la calidad a implementar (SERVQUAL)

2. Diseño del Instrumento en la Herramienta Digital Google Forms

3. Aplicación del Instrumento a una muestra de 45 personas 
4. Muestra de resultados mediantes gráficas y tablas de los resultados.

\section{Establecer el modelo de medición de la calidad a implementar (SERVQUAL)}

El presente estudio es una investigación de tipo cualitativa-cuantitativa, en la que se profundiza la empatía en los equipos de trabajo para una buena calidad de servicio que brindan las tiendas de abarrotes a los consumidores.

\section{Diseño del Instrumento en la Herramienta Digital Google Forms}

En apoyo al modelo SERVQUAL, se formuló un cuestionario en la herramienta digital forms de Google, para analizar lo que percibe el cliente; él cual cuenta con 24 ítems que se divide en las 5 "dimensiones, que nos cita: Tangibilidad, Fiabilidad, Capacidad de Respuesta, Seguridad y Empatía" (Zeithaml \& Bitner, 1985), para evaluar la percepción del cliente que responde en base a la escala Likert.

\section{Aplicación del Instrumento a un número de 45 personas}

Para determinar la calidad del servicio, se aplicó un cuestionario de 24 preguntas, en los cuales se aplicó a una muestra de 45 personas las cuales, el instrumento fue enviado a consumidores de la tienda atreves de las redes sociales usadas hoy en día como son Whatsapp, Facebook y Instagram, con el fin de conocer la percepción de que tienen de esta. El objetivo del diagnóstico es la comunidad de Escárcega que recibe el servicio de la tienda de abarrotes

\section{Muestra de resultados mediante gráficas y tablas de los resultados.}

En la tabla 2 se muestran los ítems de la dimensión de empatía, cuenta con 5 ítems, para analizar la percepción del servicio que les brinda la tienda de abarrotes, con la finalidad de medir la calidad en el servicio.
Tabla 2

Ítems SERVQUAL

\begin{tabular}{|c|c|c|c|}
\hline MEDICIÓN & $\begin{array}{l}\text { D I M EN - } \\
\text { SIÓN }\end{array}$ & No. & ITEMS \\
\hline \multirow[t]{5}{*}{$\begin{array}{l}\text { Calidad en el } \\
\text { Servicio }\end{array}$} & \multirow[t]{5}{*}{ EMPATÍA } & 20 & $\begin{array}{l}\text { ¿Se le brinda la } \\
\text { atención adecuada } \\
\text { al cliente para así } \\
\text { tener una mejor } \\
\text { comunicación? }\end{array}$ \\
\hline & & 21 & $\begin{array}{l}\text { ¿Se le brinda buen } \\
\text { equipamiento para } \\
\text { realizar sus labores } \\
\text { de trabajo? }\end{array}$ \\
\hline & & 22 & $\begin{array}{l}\text { ¿El personal de } \\
\text { servicios ofrece } \\
\text { una atención } \\
\text { personalizada a sus } \\
\text { clientes? }\end{array}$ \\
\hline & & 23 & $\begin{array}{l}\text { ¿El } \quad \text { personal } \\
\text { de servicios se } \\
\text { preocupa por los } \\
\text { mejores intereses de } \\
\text { sus clientes? }\end{array}$ \\
\hline & & 24 & $\begin{array}{l}\text { ¿El personal de } \\
\text { servicios comprende } \\
\text { las necesidades } \\
\text { específicas de sus } \\
\text { clientes? }\end{array}$ \\
\hline
\end{tabular}

\section{Resultados}

En el trabajo de investigación se muestran los siguientes resultados de acuerdo a datos obtenidos de la percepción de clientes de la tienda de abarrotes diagnosticada con respecto a la dimensión de empatía. Se muestra la importancia que tiene la empatía ya que es algo relevante en las empresas o establecimientos de abarrotes y en todo tipo de negocio indistintamente de su giro comercial, se pone en práctica nuestros resultados ya que tienen una relación objetiva a nuestra investigación sobre el desempeño de la empresa.

De acuerdo con la tabla 3, se muestran los resultados obtenidos de las encuestas con respecto a las cinco dimensiones. 


\section{Tabla 3}

Frecuencia en el nivel de satisfacción por dimensión

\begin{tabular}{|l|l|l|}
\hline $\begin{array}{l}\text { DIMENSIO - } \\
\text { NES }\end{array}$ & $\begin{array}{l}\text { NIVEL DE SATIS- } \\
\text { FACCIÓN DE } \\
\text { RE F E R E N C I A } \\
\text { "BUENO" }\end{array}$ & $\begin{array}{l}\text { FRECUENCIAS (NIVEL } \\
\text { DE SATISFACCIÓN) }\end{array}$ \\
\hline Tangibilidad & Bueno & 28 \\
\hline Fiabilidad & Bueno & 27.2 \\
\hline $\begin{array}{l}\text { Seguridad del } \\
\text { servicio }\end{array}$ & Bueno & 24.6 \\
\hline $\begin{array}{l}\text { Capacidad de } \\
\text { respuesta }\end{array}$ & Bueno & 25.4 \\
\hline Empatía & Bueno & 25 \\
\hline
\end{tabular}

\section{Figura 1}

Frecuencias Nivel de Satisfacción de Dimensiones SERVQUAL

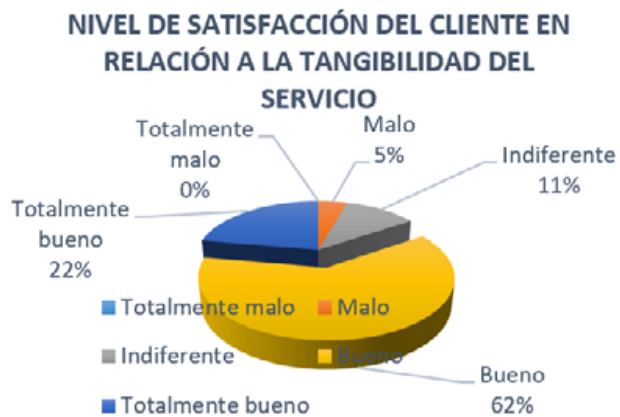

Podemos ver un análisis de los resultados en el gráfico con apoyo de la respuesta de la dimensión de tangibilidad, bueno ya que se obtuvo un $62 \%$ y con un mínimo de $5 \%$ malo, de igual forma cuenta con una frecuencia con un porcentaje bueno, se observa un $11 \%$ de individuos que tienen la percepción indiferente.
Figura 2

Resultado de diagnóstico de Tangibilidad

NIVEL DE SATISFACCIÓN DEL CLIENTE RESPECTO A LA FIABILIDAD DEL SERVICIO

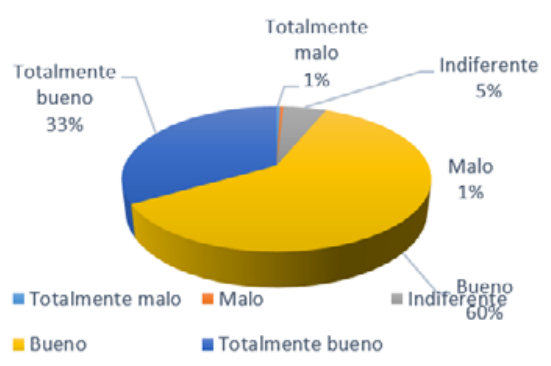

Figura 3

Resultado de diagnóstico de fiabilidad

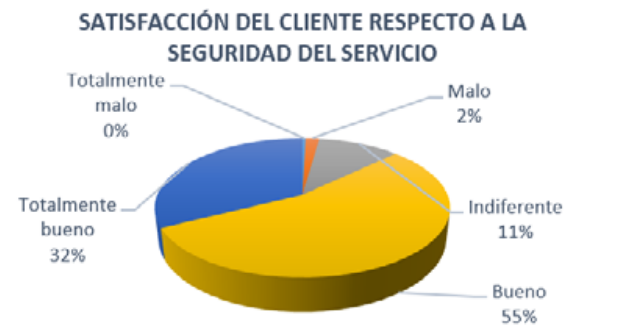

Se representa en el siguiente gráfico, los resultados obtenidos en la dimensión de fiabilidad, arroja que cuenta con un porcentaje muy alto dentro de los parámetros, de la respuesta bueno, el otro porcentaje más alto es totalmente bueno, y los porcentajes que restan respondieron que el $5 \%$ es indiferente ante la situación, y una pequeña porción lo perciben mal y totalmente malo.

\section{Capacidad de respuesta}

Se obtuvo un porcentaje regular equilibrado entre bueno y totalmente bueno, ya que fueron las respuestas con más altos porcentajes, con porcentajes de Bueno 56\% y Totalmente Bueno 32\%, un $9 \%$ respondieron de manera indiferente, y solo una minoría con un $2 \%$ y $1 \%$ respondieron de manera negativa. 


\section{Figura 4}

Resultado del Diagnóstico de Capacidad de Respuesta

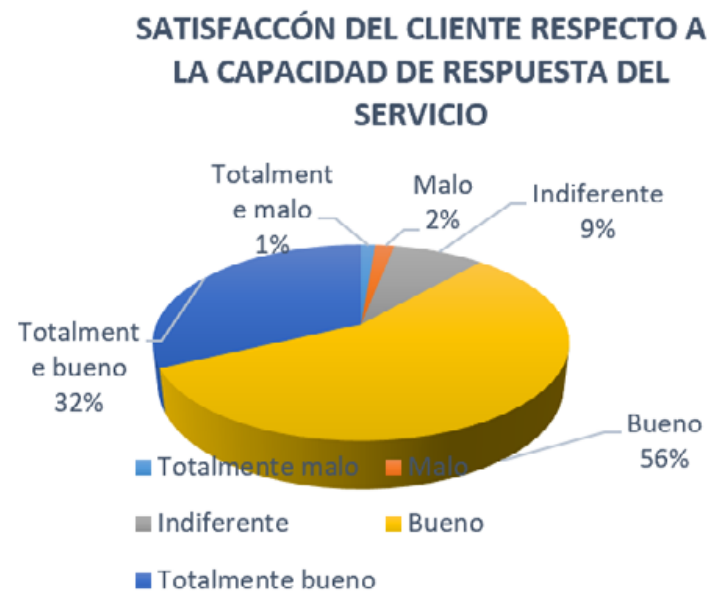

\section{Seguridad}

El análisis de la respuesta en la dimensión seguridad manifiesta un porcentaje alto en la opción de bueno con un 55\%, por consiguiente, la opción de Totalmente Bueno demuestra resultados de $32 \%$, subsiguiente el $11 \%$ respondieron de forma apática ante lo planteado en los ítems. Por último, un $2 \%$ en la respuesta de Malo en relación con la dimensión.

\section{Empatía}

Se elaboró una tabla, basándose en nuestra dimensión principal la empatía, se muestra una tabulación de datos, divida en frecuencia, porcentaje, porcentaje valido $\mathrm{y}$ porcentaje acumulado. Estas se obtuvieron en apoyo a las respuestas contestadas previamente por los consumidores del establecimiento con la escala Likert, un rango de Total Malo a Totalmente Bueno

\section{Figura 5}

Resultado de diagnóstico de seguridad

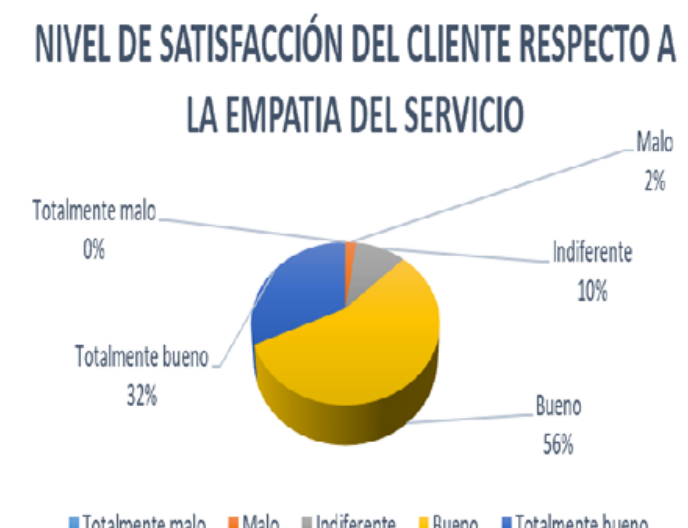

Tabla 5

Resultado de diagnóstico de empatía

\begin{tabular}{|l|l|l|l|l|}
\hline $\begin{array}{l}\text { Nivel De } \\
\text { Satisfac- } \\
\text { ción }\end{array}$ & $\begin{array}{l}\text { F r e - } \\
\text { cuencia }\end{array}$ & Porcentaje & $\begin{array}{l}\text { Porcenta- } \\
\text { je válido }\end{array}$ & $\begin{array}{l}\text { Porcentaje } \\
\text { acumulado }\end{array}$ \\
\hline $\begin{array}{l}\text { To t a 1 - } \\
\text { m e n t e } \\
\text { malo }\end{array}$ & 0 & $0 \%$ & $0 \%$ & $0 \%$ \\
\hline Malo & 1 & $2 \%$ & $2 \%$ & $2 \%$ \\
\hline $\begin{array}{l}\text { In d if e - } \\
\text { rente }\end{array}$ & 4.6 & $10 \%$ & $10 \%$ & $10 \%$ \\
\hline Bueno & 25 & $56 \%$ & $56 \%$ & $56 \%$ \\
\hline $\begin{array}{l}\text { To t a 1 - } \\
\text { m e n t e } \\
\text { bueno }\end{array}$ & 14.4 & $32 \%$ & $32 \%$ & $32 \%$ \\
\hline Total & 45 & $100 \%$ & $100 \%$ & \\
\hline
\end{tabular}

En la dimensión centrada, arroja unos resultados altos en las opciones a contestar teniendo un porcentaje de $56 \%$ en Bueno, le sigue Totalmente Bueno con un $32 \%$, y el resto del porcentaje la da lo mismo acerca del servicio que se les ofrece en el establecimiento, y último punto un $2 \%$ les parece malo acerca de la atención al cliente. 


\section{Figura 6}

Diagnóstico de resultados de empatía

FRECUENCIA DE NIVEL DE SASTIFACCIÒN "BUENO" EN LA DIMENSIONES

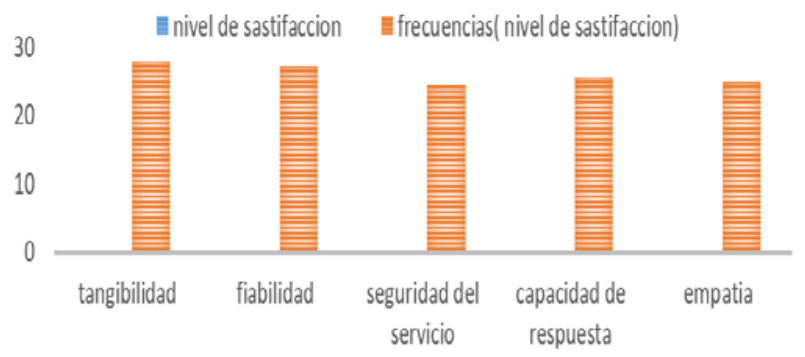

\section{Análisis}

En la tabla de frecuencia se muestran los porcentajes obtenidos de las 5 dimensiones, tomándose como referencia el nivel de satisfacción "bueno", donde se realizó la encuesta por los clientes donde el color naranja señala que es el nivel de satisfacción por cada pregunta obtenida de la dimensión correspondiente con sus respectivos resultados donde se muestra los siguientes resultados: Tangibilidad con 28\%, Fiabilidad 27.2\% la Capacidad de Respuesta $24.6 \%$ y por último $25 \%$ de empatía.

\section{Diagrama de Pareto}

\section{Tabla 6}

Diagrama de Pareto de la dimensión Empatía

\begin{tabular}{|l|l|l|l|l|}
\hline ITEM & $\begin{array}{l}\text { F R E - } \\
\text { C U E N - } \\
\text { CIA }\end{array}$ & PORCENTAJE & $\begin{array}{l}\text { \% ACUMU- } \\
\text { LADO }\end{array}$ & $80-20$ \\
\hline Q 21 & 42 & $21.54 \%$ & $21.54 \%$ & $80 \%$ \\
\hline Q 23 & 40 & $20.51 \%$ & $42.05 \%$ & $80 \%$ \\
\hline Q 24 & 40 & $20.51 \%$ & $62.56 \%$ & $80 \%$ \\
\hline Q 20 & 39 & $20.00 \%$ & $82.56 \%$ & $80 \%$ \\
\hline & & $17.44 \%$ & $100.00 \%$ & $80 \%$ \\
\hline Q .22 & 34 & & & \\
\hline TOTAL & 195 & $100.00 \%$ & & \\
\hline
\end{tabular}

\section{Figura 7}

Diagrama de Pareto de la dimensión de empatía

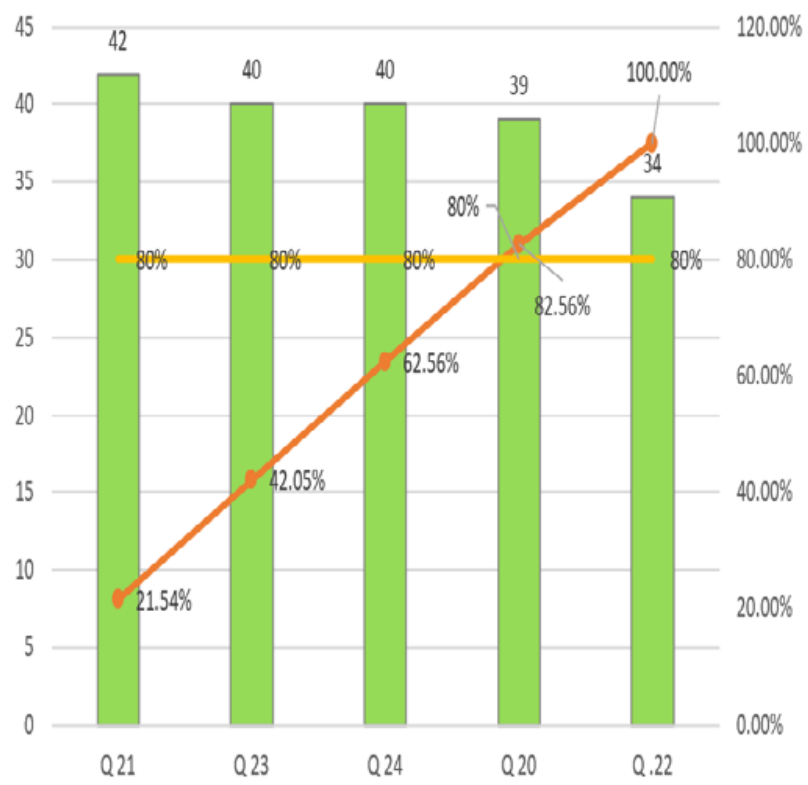

Análisis

Los siguientes datos obtenidos de este diagrama de Pareto que pertenecen la tabulación de las respuestas de la cliente basada el área de la empatía donde se mide a través de una escala Likert donde se tomaron 5 puntos donde los clientes responde la encuesta. La tabla presenta con un buen nivel de satisfacción alto. 21.54\% menciona que se brinda una atención adecuada para así tener mejor comunicación. Mientras 
$20.51 \%$ se preocupa por los mejores intereses de sus clientes. En un 20.51 también menciona que comprenden las necesidades específicas de sus clientes fue bueno. Y ya por último $20.00 \%$ comprende las necesidades específicas, que se puedan presentar ya que el servicio de la abarrotera muestra un interés en querer solucionarlos. Ya conforme a los resultados obtenidos podemos decir que hay un buen nivel de satisfacción positiva en algunos aspectos de la dimensión de empatía, no se llega a la excelencia, por no saber proporcionar soluciones al cliente en nuestros abarrotes.

Efecto: El personal de servicio no ofrece una atención personalizada.

\section{Figura 8}

\section{Causa y Efecto}

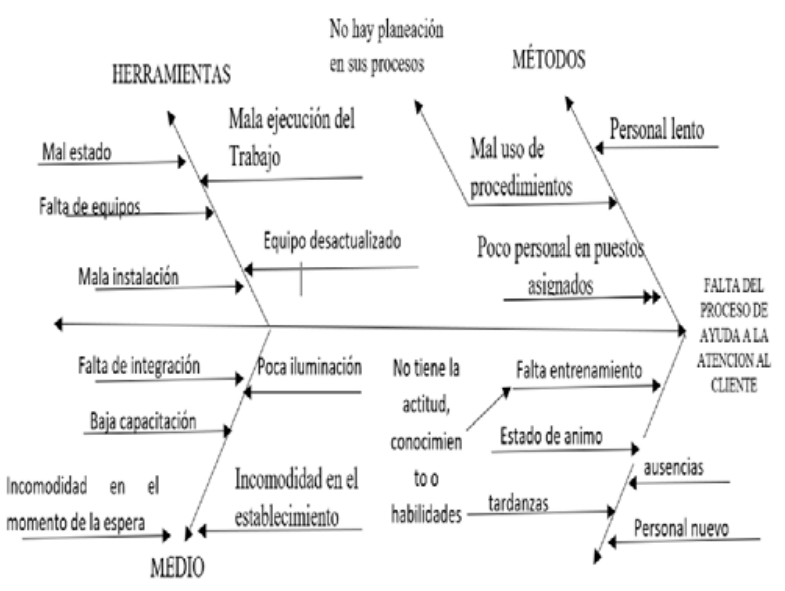

MANODE OBRA

\section{Análisis}

La herramienta aporta una mejor comprensión de un mejor panorama de los inconvenientes que causan problemas en la tienda de abarrotes, de esta forma al organizar la información que vaya centrada a la causa principal del problema, es fundamental ya que nos ayuda a determinar el punto de origen de que la empresa tenga inconveniente al brindar un buen servicio de calidad a sus clientes, se puede notar que las causas de mayor incidencia tienen en la deficiente y a si nosotros poder analizar con el método de $6 \mathrm{M}$ que nos ayuda a ver cuáles son las causas que tenemos desde como trabajamos nuestra mano de obra, métodos ya que ahí hubo una deficiencia donde la falta del personal al cliente no realizo un buen uso de procedimientos, falta de planeación entre otros que podemos observar en el diagrama.

\section{Discusión}

Esta investigación va dirigida fundamentalmente a medir la calidad en el servicio en la empresa y la empatía de los servicios de calidad que se les brinda al cliente, se menciona la gran importancia que tiene esta investigación sobre el cuestionamiento que realizado y la gran diversidad de resultados tanto buenos como malos e indiferentes entre otros, debidos a los resultados que se obtuvo nos damos cuentas de las fortalezas y debilidades que pueden tener los que conforman el equipo de la empresa y así mejora la calidad de servicio estandarizada, para llenar todos los campos de satisfacción de la persona. La mayoría de los consumidores consideran que el servicio que se brinda es totalmente bueno con un buen porcentaje casi del $100 \%$ considerando que el servicio es constantemente bueno debido a la encuesta aplicada. Ya que los 5 factores que llegamos a tocar mayormente los resultados fueron "totalmente bueno" o "bueno".

Al término de la investigación de los datos recabados acerca de la empatía y la calidad de servicio vemos su gran impacto que tiene "La competencia de empatía proporciona a las personas conciencia de las emociones, preocupaciones y necesidades de los demás. A lo largo de nuestra investigación (Vazquez, 2016) s. Tuvo como finalidad determinar el nivel de empatía y la influencia internamente en las personas que se encuentran en el ámbito laboral. El estudio fue de tipo cuantitativo con diseño descriptivo en los recursos humanos. Todavía existe la duda de una investigación futura que tenga que ver con nuestra primera investigación la investigación futura seria el cliente y sus percepciones del servicio todo el análisis que gira en torno a la calidad y la satisfacción se basa en las percepciones del cliente acerca del servicio. (Hurtado, 2011) "Uno de los retos más importantes del negocio, porque en la medida que tengamos más y mejores clientes, los volúmenes 
de venta ventas e ingresos serán mayores y por tanto, el negocio podrá sostenerse y crecer".

El concepto básico es el de "servicio percibido" tal como se analiza en el modelo de las brechas sobre la calidad en el servicio. (Sanchez, 2014) Hace referencia que la empatía es la base del sentimiento y la elección moral, pues basándose en el juicio propio es como se entiende el juicio de los otros. Considera que los humanos son animales capaces de una verdadera imitación porque no es meramente operatoria, sino que nace de dentro, de la genuina vivencia profunda y gracias a ello, puede surgir un tipo de comunidad con los otros que no es meramente impersonal, sino fraterna, que no es simplemente comunal, sino intersubjetiva (Alfredo, 2001) Conveniente ponerse en lugar de nuestros clientes para comprender lo que realmente quiere y espera cada uno de ellos. Recuerda que si como clientes esperamos nuestra plena satisfacción, como negociantes debemos estar preparados para brindar la satisfacción completa a nuestros clientes. Menciona (Derming, 1989) este carácter descriptiva está dirigida a evaluar la calidad en el servicio del sector comercio al por menor cuyos sujetos de estudio serán las tiendas de abarrotes, ultramarinos y misceláneas, situadas según (Roseello, 2008) Desde diferentes aproximaciones (e.g., evolucionista, psicofisiológica, neurológica) existe amplia evidencia científica con respecto al valor de las emociones en nuestras vidas, la finalidad de la investigación es brindar la gran importancia que se tienen la empatía en la empresa, sobre el servicio que se brinda y la buena satisfacción al cliente a si garantiza un mejor ambiente laboral a la empresa y se beneficia.

(Businessolver, 2017), señala en su investigación resultados de su empresa y el desempeño de los equipos de trabajo:

- $\quad 77 \%$ de los trabajadores estarían dispuestos a trabajar más horas en un lugar de trabajo más empático; el $60 \%$ aceptaría un salario reducido por el mismo motivo.

- $\quad$ El 92\% de los profesionales de recursos humanos indican que un entorno de trabajo empático es un factor importante para la retención de los empleados.

- $\quad$ El $80 \%$ de los millennials afirmaron que dejarían su trabajo actual si su oficina se volviera menos empática. El $66 \%$ de los Baby Boomers también compartieron este sentimiento.

Tomando en cuenta la dimensión de empatía se analizaron preguntas con un nivel alto y bajo, los consumidores perciben el servicio de buena calidad, aunque todavía quedan puntos muy débiles, debido a que en algunas respuestas que dieron, las atención personalizada es el punto débil de la empresa, ya que los empleados no dan un proceso de ayuda para el servicio. Para el establecimiento de abarrotes es importante llenar las perspectivas del cliente, con base a sus necesidades, mostrando interés y lograr los estándares que el cliente establece.

\section{Referencias bibliográficas}

Alfredo. (2001). calidad en el servicio en tiendas. México: edit.

https://www.itson.mx/publicaciones/pacioli/ Documents/no82/pacioli-82.pdf

Berry, L.L. (1989). Calidad en el servicio. Inglaterra: parasuman.

h t t p s : / / w w w. r e d a 1 y c . o r g / pdf/4717/471747525008.pdf

Bravo, R. M. (2016). Gestión de Identidad Corporativa. México: RCS.

https://produccioncientificaluz.org/index.php/ rcs/article/view/24859

Businessolver. (2017). Workplace Empathy. Obregon: Monitor.

https://info.businessolver.com/hubfs/ businessolver-workplace-empathymonitor-2017.pdf

Castillo. (2009). empatía del recurso en el servicio al cliente. Guatemala: esditmgj. 
https://www.itson.mx/publicaciones/pacioli/ Documents/no82/pacioli-82.pdf

Cohen, S. y. (1997). What makes team Works: Group effectiveness research from the shop floor to the executive. D.E: Mangement.

h t t p s : / / w w w . s c i r p.o r g / (S(1z5mqp453edsnp55rrgjct55))/ reference/References Papers. aspx?ReferenceID=962144

David. (2007). calidad en las tiendas de abarrotes. Guatemala: fut.

http://eprints.uanl.mx/362/1/1080071234.PDF

Deming. (1989). la calidad en las tiendas. Francia: crosby.

http://nulan.mdp.edu.ar/1614/1/09 administracion_calidad.pdf

Flores. (2012). empatía de recursos humanos en el servicio al cliente. Guadalajara: mex.

http://recursosbiblio.url.edu.gt/ tesiseortiz/2018/05/43/CoronadoGabriela.pdf

Frdrickson, B. (2001). The Role of Positive Emotion in Positive Psychology. Obregon: Adventure Works.

https://www.ncbi.nlm.nih.gov/pmc/articles/ PMC3122271/

García Prieto, B. y. (2003). Experiencing Diversity Conflict and Emotions in Teams . California: Internacional Review.

https://www.semanticscholar.org/paper/ Experiencing-Diversity\%2 CConflict\%2C-and-Emotions-in-GarciaPrieto-Bellard/3528129db6bb3cb065092 d5273f6461d517836ea

Hernández, C. L. (2014). Empleados Saludables y Calidad del Servicio en el Sector Sanitario. Monterrey: Diana. https://scielo.isciii.es/scielo.php?script=sci_abst ract\&pid $=$ S0212-97282014000100026

Hogan, R. (1969). Development of an Empathy Scale. Columbos: Consulting.

https://psycnet.apa.org/record/1969-12966-001

Hurtado. (2011). modalidad de calidad en el servicio. Veracruz: vezga, libro.

h t t p s : / / w w w. re d a 1 y c.org / pdf/4717/471747525008.pdf

Kozlowski, I. (2006). Enhancing the Efectiveness of Work Groups and Teams. California: Supplement.

http://bibliotecadigital.usb.edu.co/ bitstream/10819/5515/1/Equipos_alto desempe\%C3\%B1o_romero_2014.pdf

Pérez. (2005). empatía de recursos humanos en el servicio al cliente. Puebla: am editorial.

https://www.redalyc.org/pdf/167/16711589012. pdf

Quevedo, A. \&. (2008). Evaluación y propuesta de Mejoras de un modelo de Atención de Usuarios en un Servicio Público. Revista economía y administración, 71.

http://www2.udec.cl/ rea/REVISTA\%20PDF/ Rev71/art1.pdf

Rosselló, J. y. (2008). Modelos Teóricos en el Estudio Científico de la Emoción. Madrid: McGraw-Hill Interamericana.

https://dialnet.unirioja.es/servlet/ libro? código $=288543$

Rousseau, V. (2006). Teamwork Behaviors . Columbus: Research.

https://citeseerx.ist.psu.edu/viewdoc/download? doi $=10.1 .1 .474 .3973 \&$ rep $=$ rep $1 \&$ type $=p$ df

Sánchez. (2014). empatía del recurso humano. México: libros de recursos. 
http://biblio3.url.edu.gt/Tesario/2014/05/43/ Aguilar-Pahola.pdf

Vázquez. (2016). Empatía del Recurso en el Servicio al Cliente. Zacatecas: Librería Zac.

http://recursosbiblio.url.edu.gt/ tesiseortiz/2018/05/43/CoronadoGabriela.pdf

Zeithaml, V., \& Bitner, M. J. (1985). Marketing de Servicios. México: Mc Graw Hill.

https://repositorio.upn.edu.pe/ handle/11537/21122 